schizophrenia. Motor overflow, involuntary movement occurring during voluntary movement, is one such NSS found in schizophrenia.

Method: Thirty-seven participants (19 with schizophrenia, 18 controls) were tested. Participants exerted $25 \%$ and $75 \%$ of their maximal force output while overflow was monitored in the passive hand. Three transcranial magnetic stimulation protocols were designed to investigate the cortical origin of motor overflow: 1) motor cortex was stimulated unilaterally at $140 \%$ RMT; MEPs were recorded bilaterally; 2) stimulation of ispilateral hemisphere at $140 \%$ RMT was performed during motor overflow. Resulting latencies between the cMEP onset and the iSP onset were compared; 3 ) facilitated MEPs produced (through stimulation of contralateral hemisphere) during voluntary contraction and facilitated MEPs produced during motor overflow were compared. All procedures were applied to both hemispheres.

Results: Previous findings of increased motor overflow in schizophrenia compared with controls were confirmed $(P>0.05)$; neither group showed a significant difference between MEPs facilitated during voluntary movement and those facilitated during motor overflow $(P>0.05)$.

Conclusions: Results suggest that in both groups, motor overflow results from an imbalance between the transcallosal processes occurring during voluntary movement, leading to bilaterally active corticospinal tracts. Specific deficits in cortical excitability are likely to be responsible for greater overflow seen in schizophrenia.

\section{Central auditory processing deficits in patients with auditory hallucinations as shown by event-related potentials: preliminary results}

\section{H Innes-Brown', S Rossell', G Egan², D Copolov'13,4, C McKay ${ }^{5}$, T Shea ${ }^{1}$, M Wright ${ }^{1,6}$, A Sergejew ${ }^{1,7}$, K Henshall ${ }^{4}$}

Mental Health Research Institute; ${ }^{2}$ Howard Florey Institute; ${ }^{3}$ Monash University, Melbourne, Australia; ${ }^{\top}$ The University of Melbourne, Melbourne, Australia;

${ }^{5}$ Aston University; ${ }^{6}$ Alfred Hospital; and ${ }^{7}$ Maroonda Hospital

Background: It has been proposed that auditory hallucinations result from cortical or corticosubcortical disconnection. The results presented here are an initial event-related potential (ERP) exploration of data examining central auditory function and cortical connectivity.

Methods: Twenty-two controls, 26 nonhallucinating patients with psychosis and 22 currently hallucinating patients (AVH) with psychosis were recruited. ERPs to words and tones (left ear, right ear and bilaterally) were recorded during a passive listening task. Data from the left and right ear stimuli are presented here.

Results: The auditory N1 ERP was measured in two seven-channel composite regions - left temporal and right temporal. Words: N1 is enhanced contralaterally in the control group. With left words, both patient groups show reduced N1 in the right hemisphere. With right words, both patient groups show a reduction in the left hemisphere. Only AVH patients show a reduction in the ipsilateral hemisphere. Tones: No contralateral N1 enhancement is evident. With left stimuli, both patient groups show reduced N1 compared with controls in both contra- and ipsilateral hemispheres. With right stimuli, only the AVH group shows a reduction in both hemispheres.

Conclusions: The tones data confirm previous studies showing a reduced right ear advantage behaviourally in patients with schizophrenia, especially in those who hallucinate, suggesting a neurobiological origin for such behaviour. The word data suggest that more complex stimuli have a unique linguistic quality that has been more strongly lateralized. Having shown ERP differences in processing of lateralized words and tones, our next step is to look at left-right hemisphere connectivity.

\section{Self-reported depression and reduced bone mineral density in a community sample of men: Geelong Osteoporosis Study}

\section{F Jacka, J Pasco, M Henry, L Williams, M Kotowicz, G Nicholson, M Berk}

The University of Melbourne, Melbourne, Australia

Background: Previous research in psychiatric and community samples has shown reduced bone mineral density (BMD) in individuals with clinical depression and depressive symptoms, although the findings are equivocal. This study investigated the association between self-reported depression and BMD in a community sample of men aged 20-96 years enrolled in the Geelong Osteoporosis Study.

Methods: A self-report questionnaire based on DSMIV criteria was used to determine lifetime prevalence rates of depression within the study sample at baseline. Those currently taking oral glucocorticoids, testosterone or bisphosphonates were excluded $(n=23)$, resulting in a sample of 1279 men.

Results: In this sample, 155 men (12\%) reported a lifetime history of depression (LHX). There were 
no differences in age, weight, height or unadjusted BMD at the femoral neck between the depressed and nondepressed men $(P=0.08,0.34,0.41$ and 0.13 , respectively), but unadjusted BMD at the PA spine was significantly lower in those with an LHX $\left(1.254 \pm 0.187\right.$ vs. $\left.1.293 \pm 0.194 \mathrm{~g} / \mathrm{cm}^{2}, P=0.017\right)$. Age, weight and smoking-adjusted BMD were $2.8 \%$ lower at the PA spine $(1.255 \pm 0.015$ vs. $1.292 \pm$ $\left.0.006 \mathrm{~g} / \mathrm{cm}^{2}, P=0.025\right)$ and $3.0 \%$ lower at the femoral neck $\left(0.971 \pm 0.011\right.$ vs. $1.001 \pm 0.004 \mathrm{~g} / \mathrm{cm}^{2}$, $P=0.007)$ in those with an LHX compared with those nondepressed. Adjusting for selective serotonin reuptake inhibitors use did not affect these relationships.

Conclusion: These data are consistent with previous findings of diminished BMD in people with depressive disorders and symptoms and suggest that depression may be a risk factor for reduced BMD in communitydwelling adult men.

\section{Facial emotion processing in psychosis}

\section{N Joshua, A O'Regan, S Rossell}

Mental Health Research Institute of Victoria, Melbourne, Australia

Background: Investigations into facial affect processing in schizophrenia have produced variable results; some studies indicate no differences in performance, while others have shown impaired performance in patients. Variations in methodology such as the use of different stimuli, different target emotions and differing levels of task difficulty may be responsible for these inconsistent findings. The current study investigated task performance on four emotion-processing tasks in two different groups of patients with psychosis and a group of healthy controls. All four tasks used the same stimuli and target emotions but manipulated task difficulty.

Method: Age- and premorbid-IQ-matched patients with schizophrenia $(n=37)$, patients with bipolar disorder $(n=32)$ and healthy controls $(n=45)$ completed the affect discrimination, name affect, select affect and match affect subtests from the Comprehensive Affective Testing System (CATS; Froming et al. 2003).

Results: Results indicated no significant differences in performance on the affect discrimination and name affect subtests. On the select affect subtest, patients with schizophrenia were significantly impaired compared with both other groups. On the match affect subtest, patients with schizophrenia were the most impaired, followed by the bipolar group.

Conclusions: The results indicated that as task complexity increases, performance decreases, making the difference between psychosis groups and healthy controls more evident, with patients with schizophrenia showing the greatest impairment. Some subsets of the CATS may be insensitive to the subtle differences in performance between groups. Future studies should explore facial emotion processing with greater number of stimuli and include a facial processing control task.

\section{The SHADE project: self-help for alcohol/other drug use and depression \\ F Kay-Lambkin', V Carr', T Lewin', B Kelly², A Baker'}

${ }^{1}$ Centre for Mental Health Studies, University of Newcastle; and ${ }^{2}$ Centre for Rural and Remote Mental Health, Orange, Australia

Background: The co-occurrence of depression and alcohol/other drug misuse is more common than expected by chance alone. Despite this, an effective program of treatment is yet to be established for people experiencing this comorbidity. This is a concern, given rates of depression and alcohol misuse are on the increase.

Aim: This paper will report on the posttreatment alcohol/other drug- and depression-related outcomes of the SHADE project, a large-scale, multisite study of computerized psychological treatment.

Methods: SHADE participants were those with current levels of depression and current problematic use of alcohol, cannabis or amphetamines. Following an initial assessment, participants received one faceto-face case formulation session with a therapist and were subsequently randomized to receive nine sessions of SHADE therapy by a therapist, nine sessions of SHADE therapy through a computer or nine sessions of person-centered (supportive) counseling. Follow-up occurred at posttreatment, 6- and 12-month follow-up.

Results: Posttreatment results will be reported for the 250 participants recruited to the study in rural/remote and urban New South Wales.

Conclusions: Computerized treatment is not meant as a stand-alone therapy. The results from this study suggest that computer-based interventions can produce important gains for people with depression and alcohol/other drug use comorbidity. Further implications will be discussed.

\section{Duloxetine in the treatment of melancholic depression}

\section{K Kelin}

Eli Lilly Australia Pty Ltd, Australia 